\title{
Proteomic analysis of affinity captured LINE-1 macromolecular complexes
}

\author{
I. Altukhov ${ }^{1 *}$, M.S. Taylor ${ }^{2}$, K.R. Molloy 3 , P. Mita 4 , H. Jiang 5 , E.M. Adney ${ }^{4,6}$, \\ A. Wudzinska ${ }^{6}, \mathrm{~S}_{\text {. Badri }}^{7}$, D. Ischenko ${ }^{1}$, G. Eng ${ }^{2}$, K.H. Burns ${ }^{5,8}$, D. Fenyö ${ }^{4}$, \\ B.T. Chait ${ }^{3}$, D. Alexeev ${ }^{9}$, M.P. Rout ${ }^{5}$, J.D. Boeke ${ }^{4}$, J. LaCava ${ }^{4,5}$ \\ ${ }^{1}$ Moscow Institute of Physics and Technology, Dolgoprudny, Russia \\ ${ }^{2}$ Department of Pathology, Massachusetts General Hospital, Boston, United States \\ ${ }^{3}$ Laboratory of Mass Spectrometry and Gaseous Ion Chemistry, The Rockefeller University, \\ New York, United States \\ ${ }^{4}$ Department of Biochemistry and Molecular Pharmacology, Institute for Systems Genetics, \\ NYU Langone Health, New York, United States \\ ${ }^{5}$ Laboratory of Cellular and Structural Biology, The Rockefeller University, New York, United States \\ ${ }^{6}$ McKusick-Nathans Institute of Genetic Medicine, Johns Hopkins University School of Medicine, \\ Baltimore, United States \\ ${ }^{7}$ Department of Pathology, NYU Langone Health, New York, United States \\ ${ }^{8}$ Department of Pathology, Johns Hopkins University School of Medicine, Baltimore, United States \\ ${ }^{9}$ Novosibirsk State University, Novosibirsk, Russia \\ *e-mail: ilya.altukhov@gmail.com
}

Key words: protein-protein interactions, protein complex, mass-spectrometry, LINE-1, retrotransposon, data analysis

Motivation and Aim: Long Interspersed Nuclear Element-1 (LINE-1, L1) is a mobile genetic element active in human genomes. L1-encoded ORF1 and ORF2 proteins bind L1 RNAs, forming ribonucleoproteins (RNPs). These RNPs interact with diverse host proteins, some repressive and others required for the L1 lifecycle. In a never-ending battle, our cells have been fighting to keep LINE-1 and its ancestors from replicating, and so evolved various defense mechanisms. Yet, LINE-1 has learned to circumvent these barriers, and continues to replicate and cause disease. Our understanding of these defenses and of how LINE-1 evades them is limited.

Methods and Algorithms: Using differential affinity purifications, quantitative mass spectrometry, and next generation RNA sequencing, we have characterized the proteins and nucleic acids associated with distinctive, enzymatically active L1 macromolecular complexes.

Results: We described a cytoplasmic intermediate that we hypothesize to be the canonical ORF1p/ORF2p/L1-RNA-containing RNP, and we described a nuclear population containing ORF2p, but lacking ORF1p, which likely contains host factors participating in target-primed reverse transcription. We additionally explored proteomes associated with catalytically-inactivated ORF2p point mutants and monitored the rates of protein exchange from L1 macromolecules in vitro. Taken together, our data support the existence of a variety of putative L1-related protein complexes.

Conclusion: Custom computer code written in the $\mathrm{R}$ programming language was used in the analysis of mass spectrometry and RNA sequencing data; it has been published at https://github.com/elifesciences-publications/altukhov-line-1. 